\section{International Scientific Journal Theoretical \& Applied Science}

p-ISSN: 2308-4944 (print) e-ISSN: 2409-0085 (online)

Year: $2016 \quad$ Issue: 11 Volume: 43

Published: $30.11 .2016 \quad$ http://T-Science.org

SECTION 2. Applied mathematics. Mathematical modeling.
Alexandr Nikolayevich Shevtsov candidate of technical sciences, member of PILA (USA), member of European Academy of Natural History (UK), corresponding member of the Kazakhstan National Academy of Natural Sciences, President of International Academy TAS, Department of «Mathematics», Deputy Director on Science of faculty of information technologies, automation and telecommunications, Taraz state University named after M.Kh. Dulati, Kazakhstan Shev_AlexXXXX@mail.ru

\title{
ON SOME PROPERTIES OF THREE CONSECUTIVE NUMBERS
}

Abstract: The article considers the sequence of three natural numbers, properties and regularities for generalization to $n$ dimensional case extent in the calculation of the coefficient of proportionality for equality. The convergence coefficient during the growth of the initial point of reference and extent.

Key words: sequence, integer, convergence

Language: Russian

Citation: Shevtsov AN (2016) ON SOME PROPERTIES OF THREE CONSECUTIVE NUMBERS. ISJ Theoretical \& Applied Science, 11 (43): 177-185.

Soi: http://s-o-i.org/1.1/TAS-11-43-33 Doi: crossef http://dx.doi.org/10.15863/TAS.2016.11.43.33

\section{О НЕКОТОРЫХ СВОЙСТВАХ ТРЕХ ПОСЛЕДОВАТЕЛЬНЫХ ЧИСЕЛ}

Аннотация: В статье рассматриваются последовательность из трех натуральных чисел, ее свойства и закономерности для обобщения на п мерный случай степени, при вычислении коэффициента пропорциональности для равенства. Показана сходимость коэффициента при росте начальной точки отсчета и степени.

Ключевые слова: последовательность, натуральное число, сходимость.

\section{Введение}

Рассмотрим выражение состоящее из трех последовательных чисел $31+33=2 * 32$. Очевидно, что при изменении начальной точки отсчета этих последовательных чисел само выражение всегда остается верным, т.е.:

$$
\begin{aligned}
& \begin{array}{l}
1+3=2 * 2 \\
10+12=2 * 11 \\
4444+4446=2 * 4445
\end{array} \\
& \text { и т.д. }
\end{aligned}
$$

При рассмотрении выражения $\sqrt{1}+\sqrt{3}<2 \sqrt{2}$ очевидно получим знак меньше

$$
\begin{aligned}
& \operatorname{evalf}(\operatorname{sqrt}(1)+\operatorname{sqrt}(3)) ; \\
& \operatorname{evalf}(2 * \operatorname{sqrt}(2)) ;
\end{aligned}
$$

При рассмотрении выражения $\sqrt[3]{1}+\sqrt[3]{3}<2 \sqrt[3]{2}$ знак меньше сохранится evalf $\left(1^{\wedge}(1 / 3)+3^{\wedge}(1 / 3)\right)$; evalf $\left(2^{*}\left(2^{\wedge}(1 / 3)\right)\right)$;

Увеличим промежуток
for i from 1 to 50 do
evalf $\left(1^{\wedge}(1 / \mathrm{i})+3^{\wedge}(1 / \mathrm{i})\right)<\operatorname{evalf}\left(2^{*}\left(2^{\wedge}(1 / \mathrm{i})\right)\right)$;
od;
\begin{tabular}{|l|l|}
\hline $\mathrm{i}=1$ & $4=4$ \\
$\mathrm{i}=2$ & $2.732050808<2.828427124$ \\
$\mathrm{i}=3$ & $2.442249570<2.519842100$ \\
$\mathrm{i}=4$ & $2.316074013<2.378414230$ \\
$\mathrm{i}=5$ & $2.245730940<2.297396710$ \\
$\mathrm{i}=6$ & $2.200936955<2.244924096$ \\
$\mathrm{i}=7$ & $2.169930813<2.208179028$ \\
$\mathrm{i}=8$ & $2.147202690<2.181015466$ \\
$\mathrm{i}=9$ & $2.129830964<2.160119478$ \\
$\mathrm{i}=10$ & $2.116123174<2.143546926$ \\
$\ldots$ & $\ldots$ \\
$\mathrm{i}=50$ & $2.022215413<2.027918960$ \\
\end{tabular}

Обобщим данное выражение и напишем общую формулу

$$
\sqrt[n]{x+1}+\sqrt[n]{x+3}=k_{n} \sqrt[n]{x+2}
$$

где

x - начальная точка отсчета,

$\mathrm{n}$ - порядок, 


\begin{tabular}{|c|c|c|c|c|c|c|}
\hline Impact Factor: & $\begin{array}{l}\text { ISRA (India) } \\
\text { ISI (Dubai, UAE } \\
\text { GIF (Australia) } \\
\text { JIF }\end{array}$ & $\begin{array}{r}=1.344 \\
=0.829 \\
=0.564 \\
=1.500\end{array}$ & $\begin{array}{l}\text { SIS (USA) } \\
\text { PИНЦ (Russia) } \\
\text { ESJI (KZ) } \\
\text { SJIF (Morocco) }\end{array}$ & $\begin{array}{l}=0.912 \\
=0.234 \\
=1.042 \\
=2.031\end{array}$ & $\begin{array}{l}\text { ICV (Poland) } \\
\text { PIF (India) } \\
\text { IBI (India) }\end{array}$ & $\begin{array}{l}=6.630 \\
=1.940 \\
=4.260\end{array}$ \\
\hline
\end{tabular}

$k_{n}$ - коэффициент пропорциональности.

Тогда выражая

пропорциональности получим:

$k_{n}=\frac{\sqrt[n]{x+1}+\sqrt[n]{x+3}}{\sqrt[n]{x+2}}$

или

$$
k_{n}=\sqrt[n]{\frac{x+1}{x+2}}+\sqrt[n]{\frac{x+3}{x+2}}
$$

\section{Материалы и методы}

Выразим данный коэффициент $k_{n}$ на Maple и построим график функции $k_{n}$.

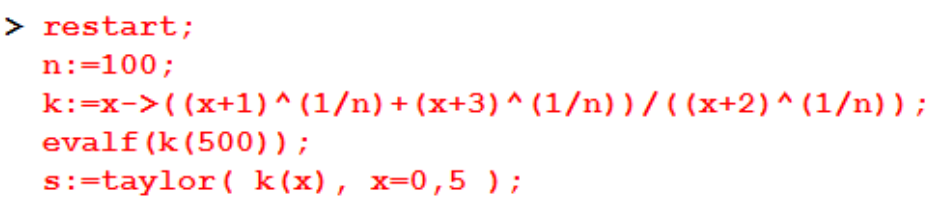

Разлагая в ряд Тейлора, получим

$$
\begin{aligned}
& s:=\frac{1}{2}\left(1+3^{1 / 100}\right) 2^{\frac{99}{100}}+\left(-\frac{1}{400}\left(1+3^{1 / 100}\right) 2^{\frac{99}{100}}+\frac{1}{2} \% 1\right) x+ \\
& \left(-\frac{1}{400} \% 1+\frac{101}{160000}\left(1+3^{1 / 100}\right) 2^{\frac{99}{100}}+\frac{1}{2}\left(-\frac{11}{20000} 3^{1 / 100}-\frac{99}{20000}\right) 2^{\frac{99}{100}}\right) x^{2}+\left(\frac{101}{160000} \% 1\right. \\
& -\frac{1}{400}\left(-\frac{11}{20000} 3^{1 / 100}-\frac{99}{20000}\right) 2^{\frac{99}{100}}-\frac{6767}{32000000}\left(1+3^{1 / 100}\right) 2^{\frac{99}{100}} \\
& \left.+\frac{1}{2}\left(\frac{2189}{18000000} 3^{1 / 100}+\frac{6567}{2000000}\right) 2^{\frac{99}{100}}\right) x^{3}+\left(\frac{1}{2}\left(-\frac{1963533}{800000000}-\frac{654511}{21600000000} 3^{1 / 100}\right) 2^{\frac{99}{100}}\right. \\
& -\frac{6767}{32000000} \% 1+\frac{2036867}{25600000000}\left(1+3^{1 / 100}\right) 2^{\frac{99}{100}}+\frac{101}{160000}\left(-\frac{11}{20000} 3^{1 / 100}-\frac{99}{20000}\right) 2^{\frac{99}{100}} \\
& \left.-\frac{1}{400}\left(\frac{2189}{18000000} 3^{1 / 100}+\frac{6567}{2000000}\right) 2^{\frac{99}{100}}\right) x^{4}+O\left(x^{5}\right) \\
& \% 1=\left(\frac{1}{300} 3^{1 / 100}+\frac{1}{100}\right) 2^{\frac{99}{100}} \\
& >\text { s:=evalf (simplify }(s)) \text {; } \\
& s:=1.997155378+.003292024341 x-.003013022780 x^{2}+.002582355946 x^{3}-.002179512808 x^{4}+\mathrm{O}\left(x^{5}\right)
\end{aligned}
$$




\begin{tabular}{l|lr|ll|ll} 
& ISRA (India) & $=\mathbf{1 . 3 4 4}$ & SIS (USA) & $=\mathbf{0 . 9 1 2}$ & ICV (Poland) & $=\mathbf{6 . 6 3 0}$ \\
Impact Factor: & ISI (Dubai, UAE) $=\mathbf{0 . 8 2 9}$ & PUHL (Russia) $=\mathbf{0 . 2 3 4}$ & PIF (India) & $=\mathbf{1 . 9 4 0}$ \\
& GIF (Australia) & $\mathbf{0 . 5 6 4}$ & ESJI (KZ) & $=\mathbf{1 . 0 4 2}$ & IBI (India) & $\mathbf{= 4 . 2 6 0}$ \\
& JIF & $\mathbf{1 . 5 0 0}$ & SJIF (Morocco) $=\mathbf{2 . 0 3 1}$ & & \\
\hline
\end{tabular}

restart;

$\mathrm{k}:=(\mathrm{x}, \mathrm{n})->\left((\mathrm{x}+1)^{\wedge}(1 / \mathrm{n})+(\mathrm{x}+3)^{\wedge}(1 / \mathrm{n})\right) /\left((\mathrm{x}+2)^{\wedge}(1 / \mathrm{n})\right) ;$

evalf $(\mathrm{k}(10,10))$;

$$
k:=(x, n) \rightarrow \frac{(x+1)^{\left(\frac{1}{n}\right)}+(x+3)^{\left(\frac{1}{n}\right)}}{1.999372998}
$$

$\operatorname{plot} 3 d(k(x, n), x=1 \ldots 10, n=1 \ldots 10)$;

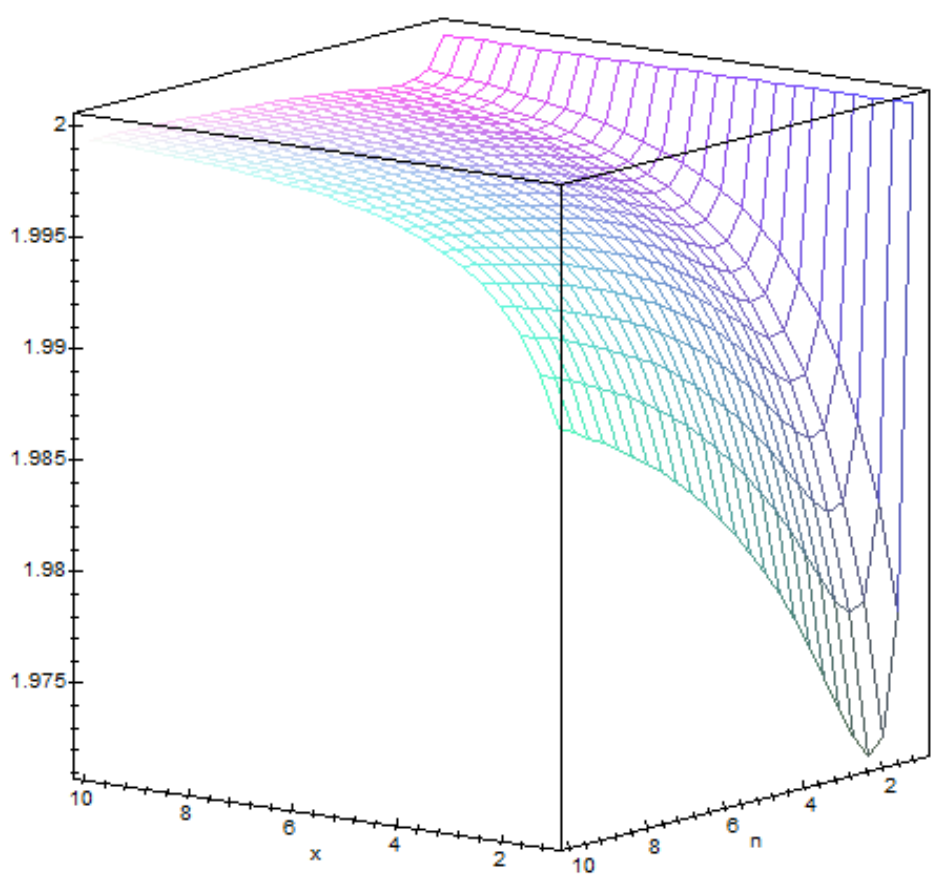

При возрастании х или n будем отмечать сходимость выражения к числу 2. 


\begin{tabular}{l|lr|ll|ll} 
& ISRA (India) & $=\mathbf{1 . 3 4 4}$ & SIS (USA) & $=\mathbf{0 . 9 1 2}$ & ICV (Poland) & $=\mathbf{6 . 6 3 0}$ \\
Impact Factor: & ISI (Dubai, UAE) $=\mathbf{0 . 8 2 9}$ & PUHU (Russia) $=\mathbf{0 . 2 3 4}$ & PIF (India) & $=\mathbf{1 . 9 4 0}$ \\
& GIIF (Australia) & $\mathbf{0 . 5 6 4}$ & ESJI (KZ) & $=\mathbf{1 . 0 4 2}$ & IBI (India) & $=\mathbf{4 . 2 6 0}$ \\
& JIIF & $\mathbf{1 . 5 0 0}$ & SJIF (Morocco) $=\mathbf{2 . 0 3 1}$ & & \\
\hline
\end{tabular}

$\operatorname{plot3d}(k(x, n), x=1 \ldots 1000, n=1 \ldots 1000)$;

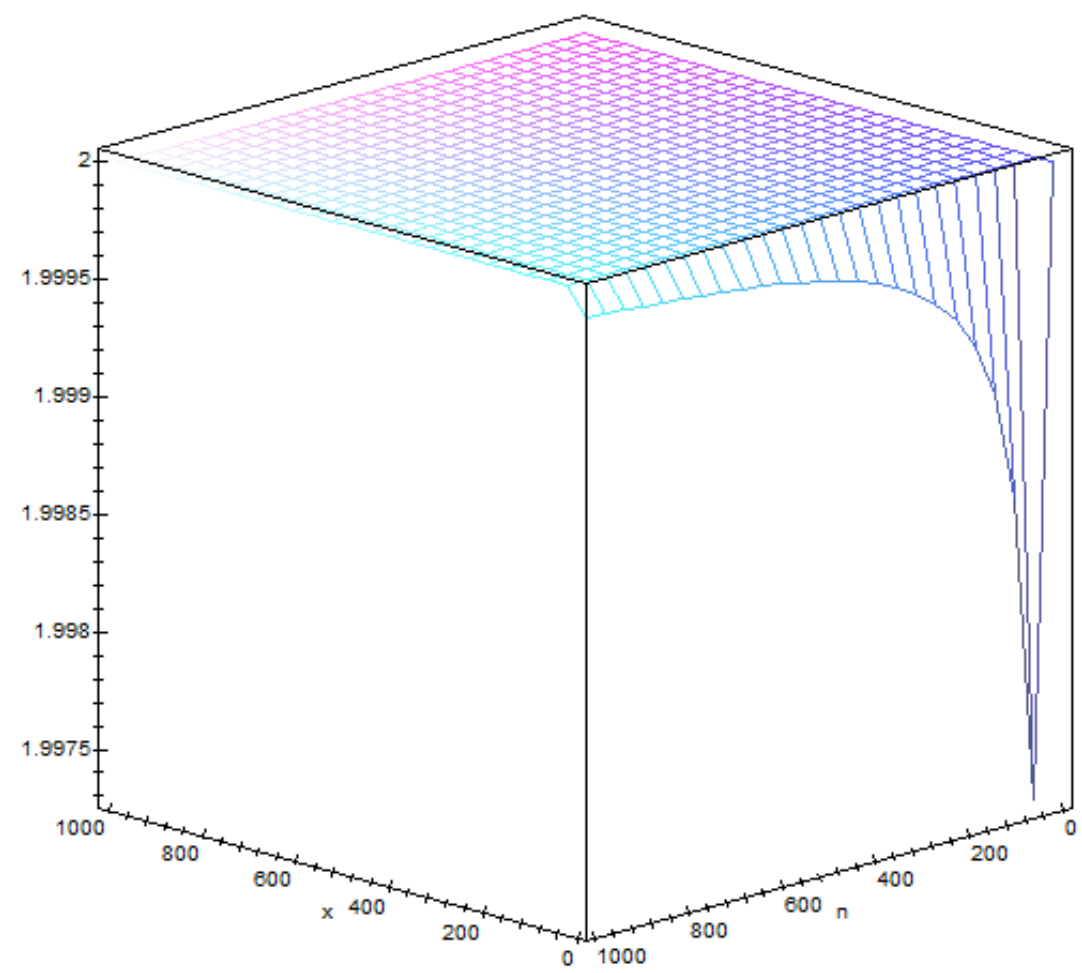

Если вместо корня использовать степень, получим

restart;

$\mathrm{k}:=(\mathrm{x}, \mathrm{n})->\left((\mathrm{x}+1)^{\wedge}(\mathrm{n})+(\mathrm{x}+3)^{\wedge}(\mathrm{n})\right) /\left((\mathrm{x}+2)^{\wedge}(\mathrm{n})\right)$;

evalf $(k(10,10))$;

$$
\begin{gathered}
k=(x, n) \rightarrow \frac{(x+1)^{n}+(x+3)^{n}}{(x+2)^{n}} \\
2.645395496
\end{gathered}
$$

plot3d $(k(x, n), x=1 \ldots 10, n=1 \ldots 10)$; 


\begin{tabular}{|c|c|c|c|c|c|c|}
\hline Impact Factor: & $\begin{array}{l}\text { ISRA (India) } \\
\text { ISI (Dubai, UAE } \\
\text { GIF (Australia) } \\
\text { JIF }\end{array}$ & $\begin{array}{r}=1.344 \\
=0.829 \\
=0.564 \\
=1.500\end{array}$ & $\begin{array}{l}\text { SIS (USA) } \\
\text { PИНЦ (Russia) } \\
\text { ESJI (KZ) } \\
\text { SJIF (Morocco) }\end{array}$ & $\begin{array}{l}=0.912 \\
=0.234 \\
=1.042 \\
=2.031\end{array}$ & $\begin{array}{l}\text { ICV (Poland) } \\
\text { PIF (India) } \\
\text { IBI (India) }\end{array}$ & $\begin{array}{l}=6.630 \\
=1.940 \\
=4.260\end{array}$ \\
\hline
\end{tabular}

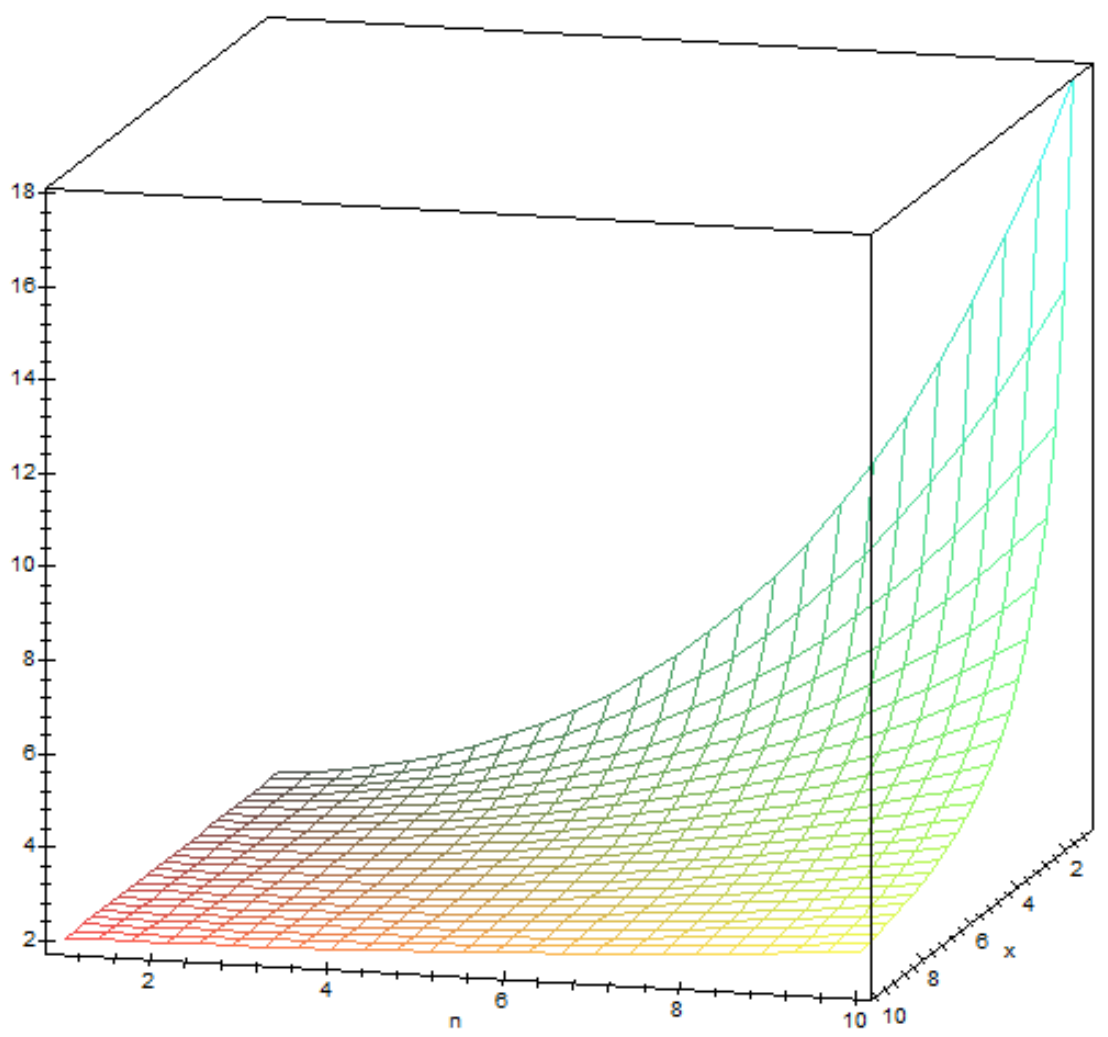

plot3d (k (x, n), $x=1 \ldots 1000, n=1 \ldots 1000)$;

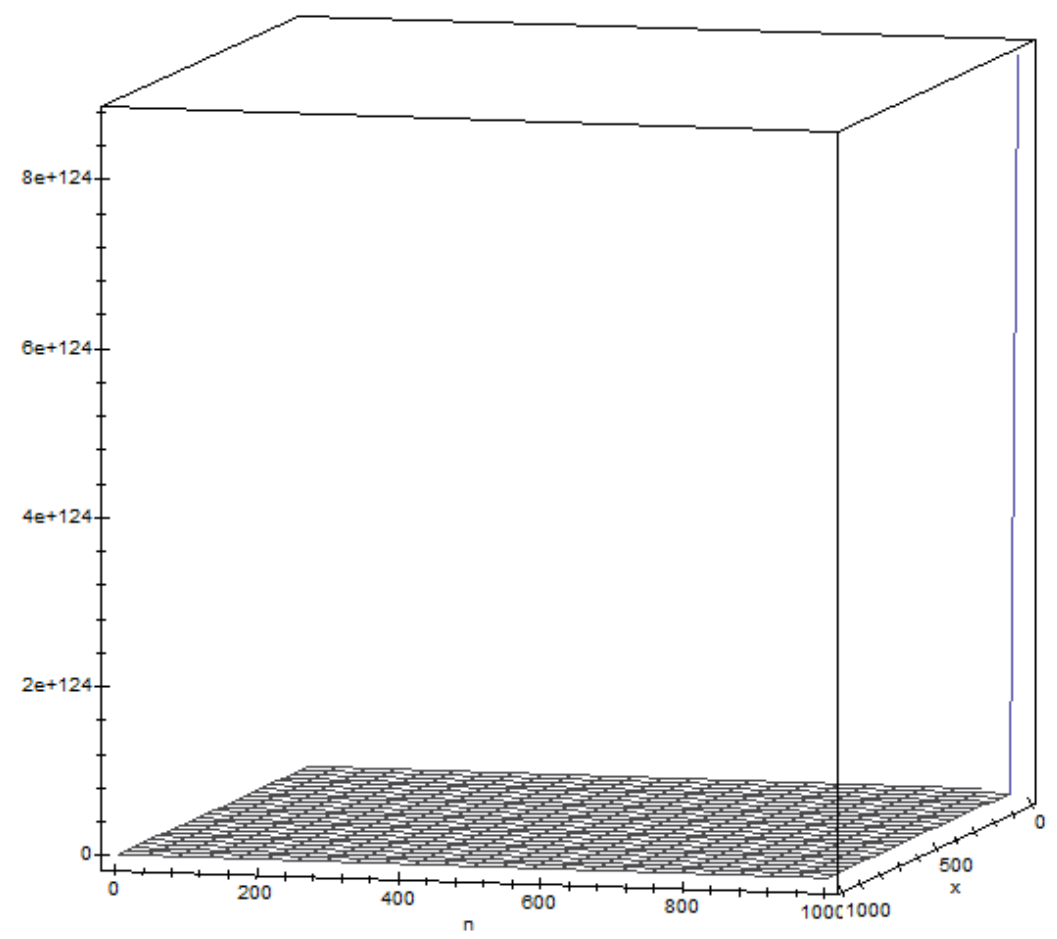

ISPC Education and Innovation, 


\begin{tabular}{|c|c|c|c|c|c|c|}
\hline Impact Factor: & $\begin{array}{l}\text { ISRA (India) } \\
\text { ISI (Dubai, UAE } \\
\text { GIF (Australia) } \\
\text { JIF }\end{array}$ & $\begin{array}{r}=1.344 \\
=0.829 \\
=0.564 \\
=1.500\end{array}$ & $\begin{array}{l}\text { SIS (USA) } \\
\text { PИНЦ (Russia) } \\
\text { ESJI (KZ) } \\
\text { SJIF (Morocco) }\end{array}$ & $\begin{array}{l}=0.912 \\
=0.234 \\
=1.042 \\
=2.031\end{array}$ & $\begin{array}{l}\text { ICV (Poland) } \\
\text { PIF (India) } \\
\text { IBI (India) }\end{array}$ & $\begin{array}{l}=6.630 \\
=1.940 \\
=4.260\end{array}$ \\
\hline
\end{tabular}

restart;

$\mathrm{k}:=(\mathrm{x}, \mathrm{n})->\left((\mathrm{x}+1)^{\wedge}(\mathrm{n})+(\mathrm{x}+3)^{\wedge}(\mathrm{n})\right) /\left((\mathrm{x}+2)^{\wedge}(\mathrm{n})\right)$;

$\mathrm{k} 2:=(\mathrm{x}, \mathrm{n})->\left((\mathrm{x}+1)^{\wedge}(1 / \mathrm{n})+(\mathrm{x}+3)^{\wedge}(1 / \mathrm{n})\right) /\left((\mathrm{x}+2)^{\wedge}(1 / \mathrm{n})\right)$;

$$
\begin{gathered}
k:=(x, n) \rightarrow \frac{(x+1)^{n}+(x+3)^{n}}{(x+2)^{n}} \\
k 2=(x, n) \rightarrow \frac{(x+1)^{\left(\frac{1}{n}\right)}+(x+3)^{\left(\frac{1}{n}\right)}}{\left(\frac{1}{n}\right)}
\end{gathered}
$$

$\operatorname{plot} 3 d(\{k(x, n), k 2(x, n)\}, x=1 \ldots 10, n=1 \ldots 10$, axes=boxed, orientation= $[60,70])$;

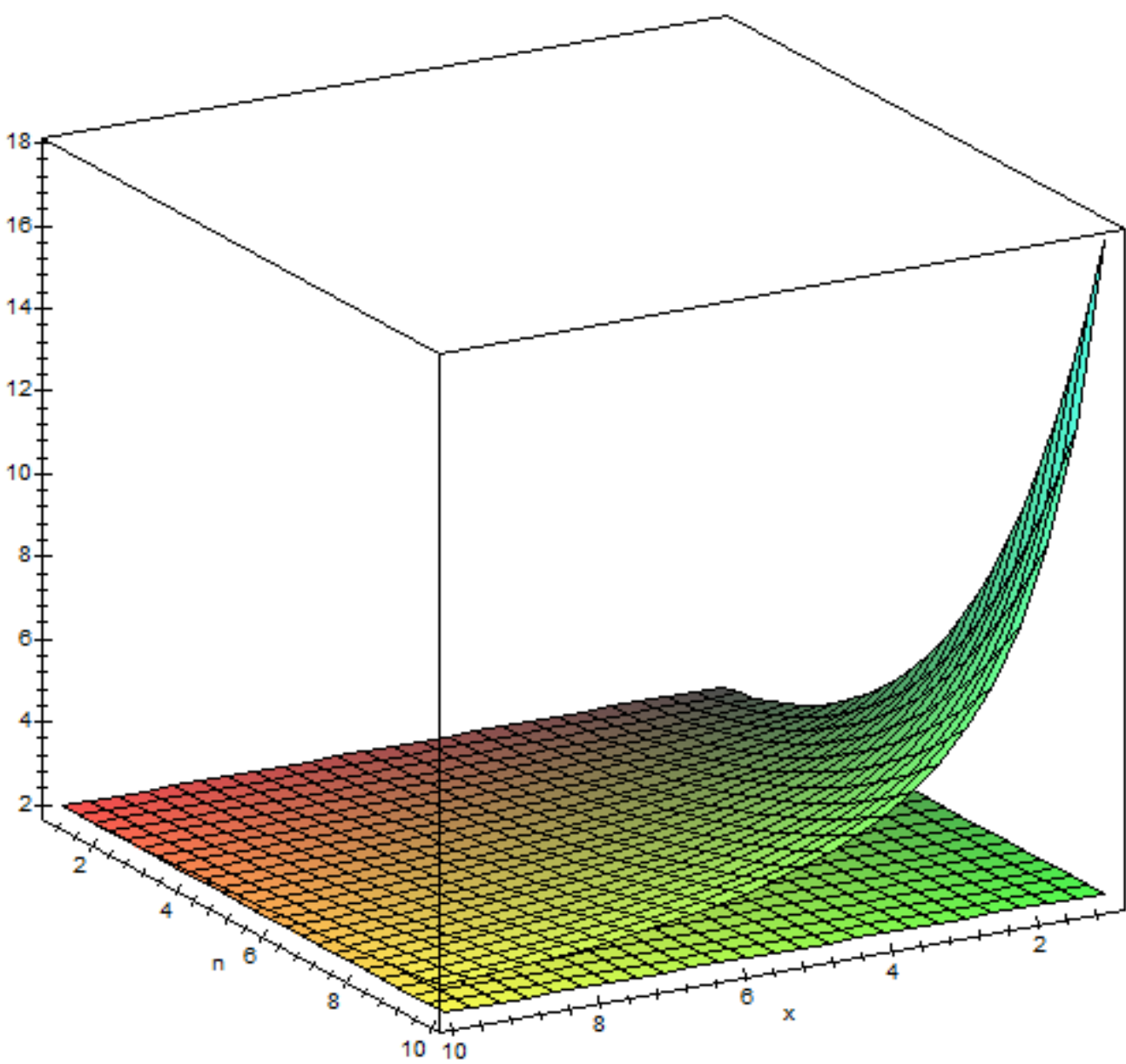

$\operatorname{plot} 3 d(\{k(x, n), k 2(x, n)\}, x=-5 \ldots 5, n=1 \ldots 10$, axes=boxed, orientation $=[60,70])$; 


\begin{tabular}{l|lr|ll|ll} 
& ISRA (India) & $=\mathbf{1 . 3 4 4}$ & SIS (USA) & $=\mathbf{0 . 9 1 2}$ & ICV (Poland) & $=\mathbf{6 . 6 3 0}$ \\
Impact Factor: & ISI (Dubai, UAE) & $=\mathbf{0 . 8 2 9}$ & PUHЦ (Russia) & $=\mathbf{0 . 2 3 4}$ & PIF (India) & $=\mathbf{1 . 9 4 0}$ \\
& GIF (Australia) & $=\mathbf{0 . 5 6 4}$ & ESJI (KZ) & $=\mathbf{1 . 0 4 2}$ & IBI (India) & $=\mathbf{4 . 2 6 0}$ \\
& JIF & $=\mathbf{1 . 5 0 0}$ & SJIF (Morocco) & $\mathbf{2 . 0 3 1}$ & & \\
\hline
\end{tabular}

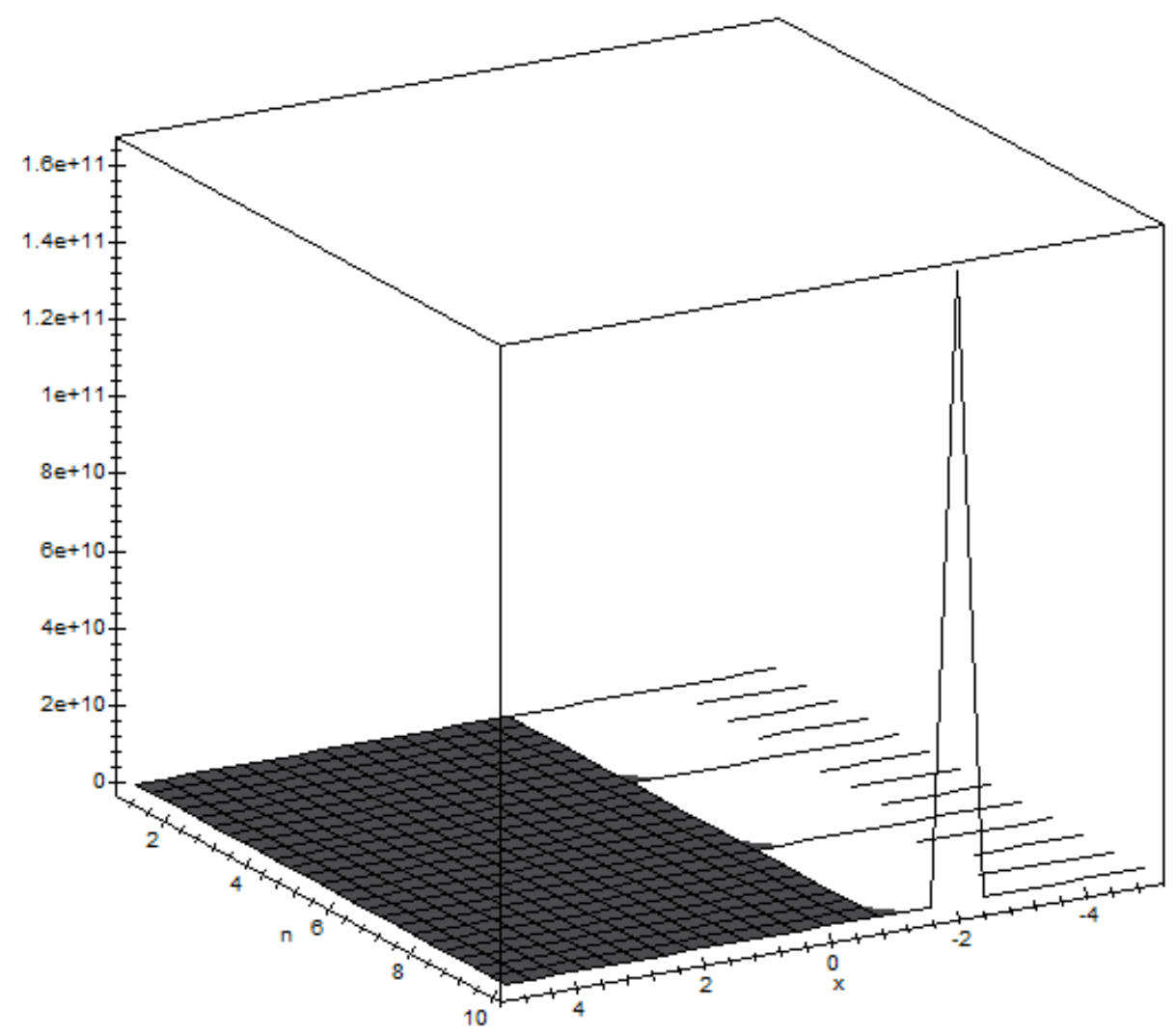

$\operatorname{plot} 3 d(\{k(x, n), k 2(x, n)\}, x=-10 \ldots 0, n=1 \ldots 9$, axes=boxed, orientation= $[60,70])$;

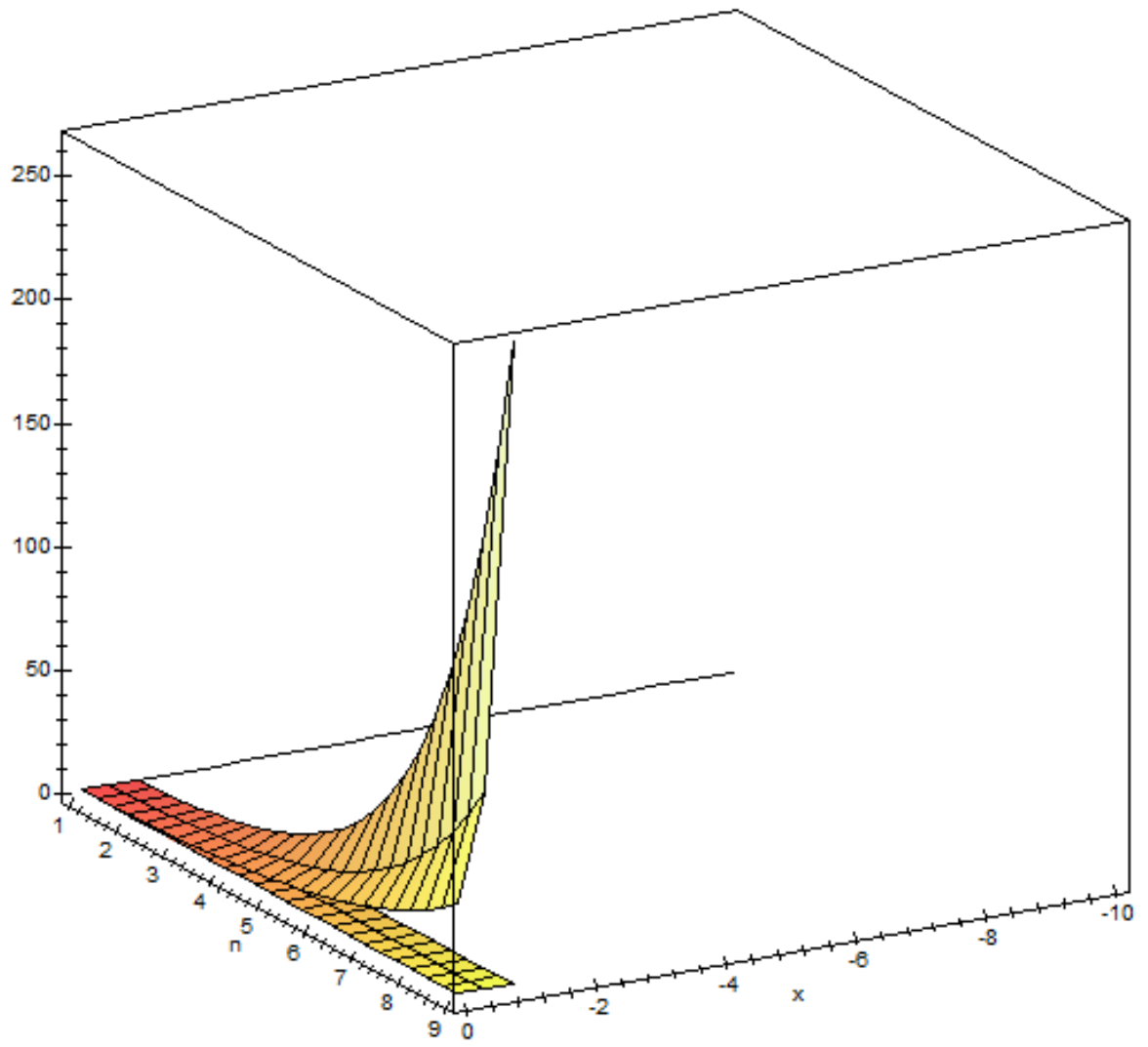

ISPC Education and Innovation, 


\begin{tabular}{|c|c|c|c|c|c|c|}
\hline Impact Factor: & $\begin{array}{l}\text { ISRA (India) } \\
\text { ISI (Dubai, UAE } \\
\text { GIF (Australia) } \\
\text { JIF }\end{array}$ & $\begin{array}{r}=1.344 \\
=0.829 \\
=0.564 \\
=1.500\end{array}$ & $\begin{array}{l}\text { SIS (USA) } \\
\text { PИНЦ (Russia) } \\
\text { ESJI (KZ) } \\
\text { SJIF (Morocco) }\end{array}$ & $\begin{array}{l}=0.912 \\
=0.234 \\
=1.042 \\
=2.031\end{array}$ & $\begin{array}{l}\text { ICV (Poland) } \\
\text { PIF (India) } \\
\text { IBI (India) }\end{array}$ & $\begin{array}{l}=6.630 \\
=1.940 \\
=4.260\end{array}$ \\
\hline
\end{tabular}

$\operatorname{plot} 3 d(\{k(x, n), k 2(x, n)\}, x=-5 \ldots 5, n=1 \ldots 9$, axes=boxed, orientation= $[60,70])$;

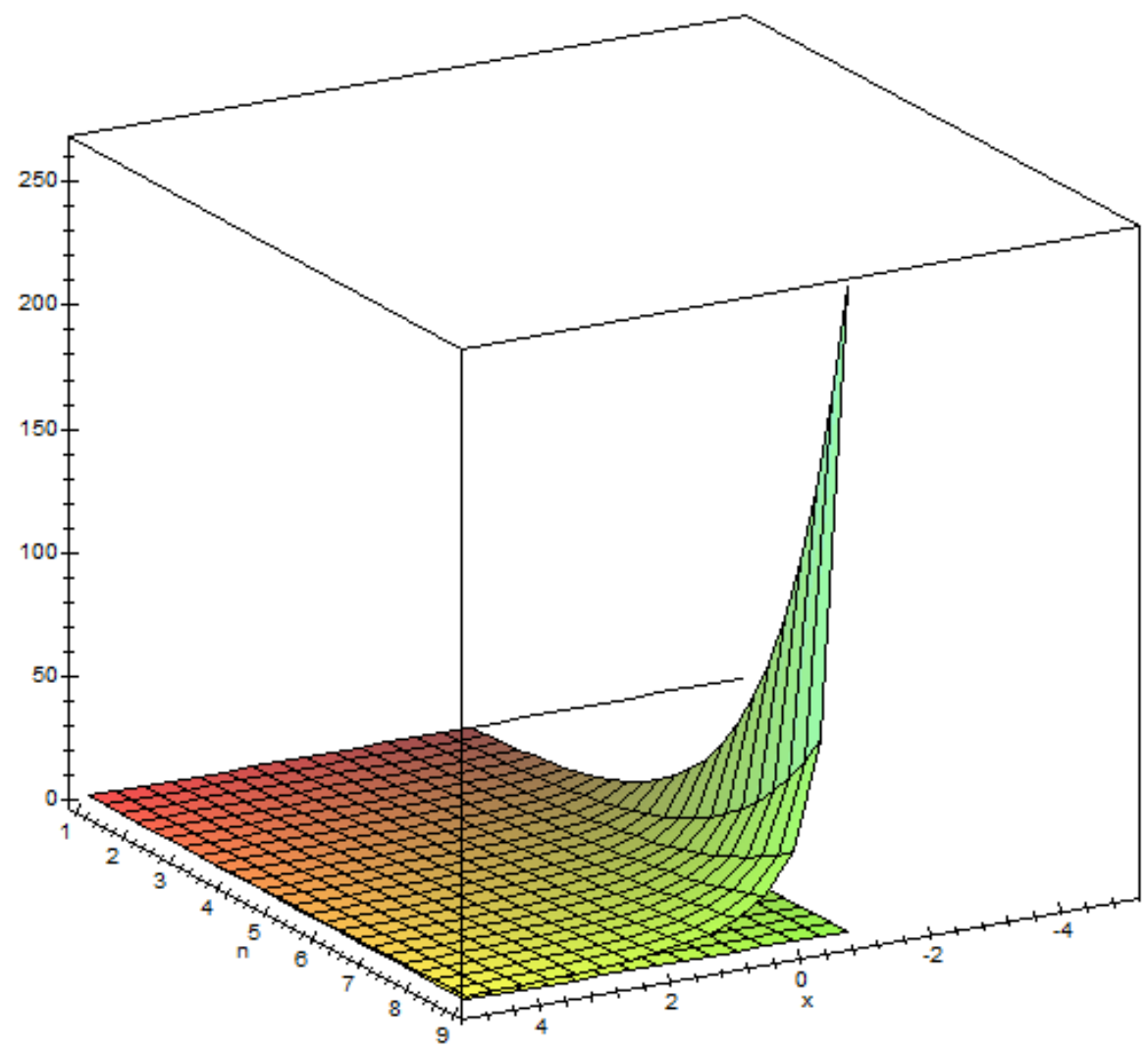

$\operatorname{plot} 3 d(\{k(x, n), k 2(x, n)\}, x=-1 \ldots 1, n=1 \ldots 9$, axes=boxed, orientation= $[60,70])$;

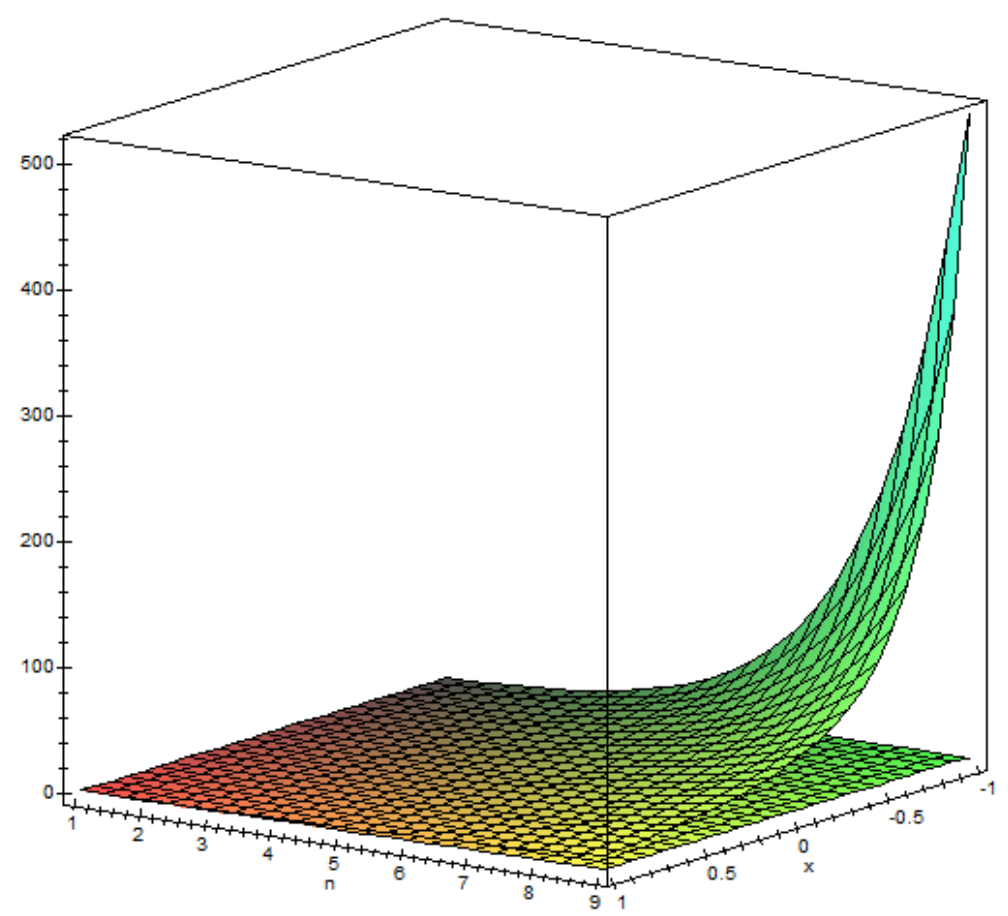

ISPC Education and Innovation, 


\begin{tabular}{|c|c|c|c|c|c|c|}
\hline Impact Factor: & $\begin{array}{l}\text { ISRA (India) } \\
\text { ISI (Dubai, UAE } \\
\text { GIF (Australia) } \\
\text { JIF }\end{array}$ & $\begin{array}{l}=1.344 \\
=0.829 \\
=0.564 \\
=1.500\end{array}$ & $\begin{array}{l}\text { SIS (USA) } \\
\text { PИНЦ (Russia } \\
\text { ESJI (KZ) } \\
\text { SJIF (Moroccc }\end{array}$ & $\begin{aligned}= & 0.912 \\
= & 0.234 \\
= & 1.042 \\
= & 2.031\end{aligned}$ & $\begin{array}{l}\text { ICV (Poland) } \\
\text { PIF (India) } \\
\text { IBI (India) }\end{array}$ & $\begin{array}{l}=6.630 \\
=1.940 \\
=4.260\end{array}$ \\
\hline
\end{tabular}

\section{Заключение}

Отметим, что первоначально задача ставилась как попытка доказать отсутствие зависимости между коэффициентов $k_{n}$ и начальной точкой $x$. Очевидно, что при $\kappa_{1}=2$ какая-либо зависимость отсутствует.

При $n \neq 1$ показана сходимость коэффициента к 2 , при росте начальной точки отсчета и степени для $x \geq-1$.

\section{References:}

1. Stephen Lynch (2010) Dynamical Systems with Applications using Maple, 2nd ed.

2. Shingareva I, Lizarraga-Celaya C (2009) Maple and Mathematica - A Problem Solving Approach for Mathematics, 2nd ed.

3. Kirsanov MN (2011) "Praktika programmirovaniya $\mathrm{v}$ sisteme Maple" M.: Izdatel'skiy dom MEI, 2011, 208p.

4. Kirsanov MN (2010) Zadachi po teoreticheskoy mekhanike $\mathrm{s}$ resheniyami $\mathrm{v}$ Maple 11. M.: Fizmatlit, 2010, 264p.

5. Korobov VI, Ochkov VF (2009) Khimicheskaya kinetika: vvedenie $\mathrm{s}$ Mathcad/Maple/MCS. M.: Goryachaya liniya-Telekom, 2009.

6. Charl'z Genri Edvards, Devid E. Penni (2007) Differentsial'nye uravneniya i kraevye zadachi: modelirovanie i vychislenie s pomoshch'yu Mathematica, Maple i MATLAB. 3-e izdanie. Kiev.: Dialektika-Vil'yams, 2007. ISBN 978-58459-1166-7.

7. Kirsanov MN (2007) Grafy v Maple. M.: Fizmatlit, 2007, 168s..

8. Alad'ev V3, Boyko VK, Rovba EA (2007) Programmirovanie i razrabotka prilozheniy v Maple. Grodno, Tallin, 2007.

9. Alad'ev VZ (2006) Osnovy programmirovaniya v Maple. Tallin, 2006..

10. Alekseev ER, Chesnokova OV (2006) Reshenie zadach vychislitel'noy matematiki v paketakh Mathcad 12, MATLAB 7, Maple 9. M: NT Press, 2006, 496s. ISBN: 5-477-00208-5.

11. Alad'ev VZ (2006) Sistemy komp'yuternoy algebry. MAPLE: Iskusstvo programmirovaniya. Binom.Laboratoriya znaniy, 2006. Annotatsiya.

12. D'yakonov VP (2004) Maple $9 \mathrm{~V}$ matematike, fizike i obrazovanii. M.: SOLON-Press, 2004.

13. Goloskokov DP (2004) Uravneniya matematicheskoy fiziki. Reshenie zadach v sisteme Maple. S-Pb: Piter, 2004.
14. D'yakonov VP (2003) Maple 8 v matematike, fizike i obrazovanii. M.: SOLON-Press, 2003

15. Sdvizhkov OA (2003) Matematika na komp'yutere: Maple 8, Solon-press, 2003.

16. Vasil'ev N (2003) Maple 8. Samouchitel', M.: Dialektika, Vil'yams, 2003. Annotatsiya.

17. Yu.Tarasevich (2003) Informatsionnye tekhnologii $\mathrm{v}$ matematike. M: SOLONPress, 2003.

18. Kirsanov M (2002) Reshebnik. Teoreticheskaya mekhanika. Fizmatlit. 2002.

19. Tan K (2001) Simvol'nyy S ++: Vvedenie v komp'yuternuyu algebru $\mathrm{s}$ ispol'zovaniem ob"ektno-orientirovannogo programmirovaniya. Mir. 2001. 